\title{
Prehospital Index provides prognosis for hospitalized patients with acute trauma
}

This article was published in the following Dove Press journal:

Patient Preference and Adherence

\author{
Hai-lin Ruan ${ }^{1, *}$ \\ Wen-han $\mathrm{Ge}^{2, *}$ \\ Jian-ping Chen' \\ Yuan-qun Zhu ${ }^{3}$ \\ Wei Huang'
}

'Department of Emergency, Fourth Affiliated Hospital of Guangxi

Medical University, Liuzhou, Guangxi, ${ }^{2}$ Department of Emergency, Huai'an Hospital Affiliated with Xuzhou Medical University, Huai'an, Jiangsu, ${ }^{3}$ Department of Neurology, Fourth Affiliated Hospital of Guangxi Medical University, Liuzhou, Guangxi, China

*These authors contributed equally to this work
Correspondence: Jian-ping Chen Department of Emergency, Fourth Affiliated Hospital of Guangxi Medical University, Liushi Road, Yufeng Qu, Liuzhou, Guangxi 545005, China

Tel +86772 38I 5284

Email drchen08@gmail.com
Objective: To evaluate the prognostic value of the Prehospital Index (PHI) for hospitalized patients with acute trauma.

Materials and methods: PHI score and the Injury Severity Score (ISS) were determined in 1,802 hospitalized patients with acute trauma. Receiver-operator characteristic (ROC) curves were used to compare the PHI and ISS in subgroups, and corresponding prediction indicators were calculated.

Results: There were significant differences in PHI score and ISS between the survival group and the death group $(Z=2.674, P=0.007)$. The area under the ROC curve was $0.871(95 \% \mathrm{CI}$ $0.855-0.886)$ for PHI score and 0.792 (95\% CI 0.773-0.811) for ISS. Optimal cutoff points to determine the risk of critical illness were PHI $\geq 4$ and ISS $\geq 22$. The sensitivity of the PHI was superior to the ISS $\left(\chi^{2}=6.975, P=0.008\right)$, but the specificity and the accuracy of the PHI and ISS showed no significant difference $(P>0.05)$.

Conclusion: The PHI is valuable in prognostic prediction of hospitalized patients with acute trauma, and it is superior to the ISS. The PHI has such advantages as being simple in operation, easy to learn, capable of reflecting conditions timely and reliably, and suitable for dynamic evaluation and screening for critical patients with trauma.

Keywords: Prehospital Index, trauma, hospitalized patient, Injury Severity Score

\section{Introduction}

Due to socioeconomic, industrial, and transport development, people are engaged in outdoor activities more frequently. Trauma has become a major threat to human health in modern society. Trauma-induced mortality has been decreasing in the past 20 years, due to progress in transport, emergency rescue, and resuscitation of wounded people. ${ }^{1,2}$ According to World Health Organization statistics, over 5 million people die from trauma worldwide each year. It is expected that by 2020 , there will be 800 trauma-related deaths worldwide. China ranks first in the world in the number of deaths related to traffic accidents. Trauma is the leading reason of death among people aged 1-44 years and ranks the third among all causes of death. ${ }^{3}$ Trauma is a serious public health issue and brings a huge burden to the society.

Severe trauma, especially multiple trauma, is usually caused by one or several factors that affect multiple sites of the body. ${ }^{4}$ Patients cannot present a medical history or cooperate with physical examination, contributing to difficult early diagnosis. Patient conditions are usually combined with various complications and infection, and may die because of delayed treatment. ${ }^{5-7}$ There are three peaks of death after severe trauma. ${ }^{8}$ The first peak occurs several seconds or minutes after trauma, which is known as death at the scene, accounting for about $50 \%$ of deaths. The second peak occurs within several hours after trauma, and is known as early death, accounting for 
about $30 \%$ of deaths. The third peak occurs 24 hours after trauma, typically in the first to fourth week after the injury and accounting for $10 \%-20 \%$ of deaths. Therefore, the prognosis of patients with severe trauma depends largely on prehospital emergency rescue. Effective first aid within 10 minutes is crucial for reducing death at the scene. Definitive treatment within 1 hour determines the prognosis of severe-trauma patients, and mortality may decrease by about $10 \%$ if the emergency rescue is timely and effective. ${ }^{9}$

One retrospective cohort study showed that lowering mortality rates in trauma patients may require reducing both the incidence of major complications and the incidence of death after major complications. ${ }^{10}$ The Prehospital Index (PHI) was developed to provide an objective prehospital scoring system for distinguishing minor-trauma patients from those requiring general or neurosurgical operative intervention within 24 hours (major trauma). ${ }^{11}$ To develop an easy and reliable method to assess hospitalized patients with trauma, improve the quality of in-hospital rescue, and reduce trauma-related deaths, in this study we assessed the prognostic value of the PHI at 24 hours before being hospitalized for patients with trauma.

\section{Materials and methods Subjects}

All patients provided written informed consent and this study was performed following the requirements of the ethics committee of the Fourth Affiliated Hospital of Guangxi Medical University. A total of 15,074 hospitalized patients with trauma from 2008 to 2011 were included. Stratified random sampling was performed to ensure that each subgroup within the population received proper representation within the sample, and $20 \%$ of cases $(3,015)$ were chosen. These cases were further screened based on criteria of initial diagnosis at our hospital, Abbreviated Injury Scale (AIS) score $\geq 3$, prehospital time $\leq 24$ hours, explicit outcomes, and intact data.

\section{Scoring}

Trauma Database version 3.0, developed by the Trauma Database Research Center of the Third Military Medical University (Chongqing, China), was used. The initial course of diseases upon admission and relevant data during the hospitalization period were recorded, including test results, surgical procedures, and final diagnoses. The data elements were coded, and the PHI and Injury Severity Score (ISS) were calculated automatically. Assessment parameters of PHI were all based on medical records upon initial admission following the scoring criteria described previously. ${ }^{11}$ AIS scores and ISS were calculated according to AIS-ISS 2005. ${ }^{12}$

\section{Statistical analysis}

All data are expressed as mean \pm SD. Measurements were compared using Student's $t$-test, and counts were compared using $\chi^{2}$ tests. Receiver-operator characteristic (ROC) curves were plotted and the area under the curve $\left(\mathrm{AUC}_{\mathrm{ROC}}\right)$ calculated. Optimal cutoff points were determined. Inferences about differences in AUC were made using Z-tests. All statistical analyses were performed using SPSS 13.0 software (SPSS Inc., Chicago, IL, USA). $P<0.05$ indicated significant difference.

\section{Results}

\section{Baseline information}

Based on stratified random sampling of 15,074 hospitalized patients with trauma, $20 \%$ of the cases $(3,015)$ were chosen, and the sampling error was estimated as $<5 \%$. Based on inclusion criteria, 1,802 hospitalized patients (1,283 males and 519 females) with trauma were eligible. They were aged $0-100$ years, with an average of $41 \pm 22$ years. The average length of hospital stay was $26 \pm 31$ days and 104 patients died, with a mortality of $5.77 \%$.

\section{$\mathrm{PHI}$ and ISS scores in patients with different prognoses}

Depending on prognosis, patients were divided into a survival group (1,698 cases) and a death group (104 cases). The PHI was $1.36 \pm 2.18$ in the survival group and $6.46 \pm 4.54$ in the death group, significantly higher in the latter $(t=11.384$, $P<0.001)$. The ISS was $13.56 \pm 6.48$ in the survival group and $21.88 \pm 7.82$ in the death group, significantly higher in the latter $(t=10.621, P<0.001)$.

\section{AUC values for $\mathrm{PHI}$ and ISS}

ROC curves were plotted for the PHI and ISS (Figure 1). $\mathrm{AUC}_{\mathrm{ROC}}$ was 0.871 for the PHI (95\% CI 0.855-0.886) and 0.792 (95\% CI $0.773-0.811)$ for the ISS, which indicated a significant difference between the two groups $(Z=2.674$, $P=0.007)$. Optimal cutoff points were determined based on Youden's index. Cutoff values for diagnosing potential critical diseases among hospitalized patients with acute trauma were PHI $\geq 4$ and ISS $\geq 22$, respectively.

\section{Comparison of evaluation indices of $\mathrm{PHI}$ and ISS}

Sensitivity, specificity, accuracy, Youden's index, falsepositive rate, false-negative rate, positive predictive value, 


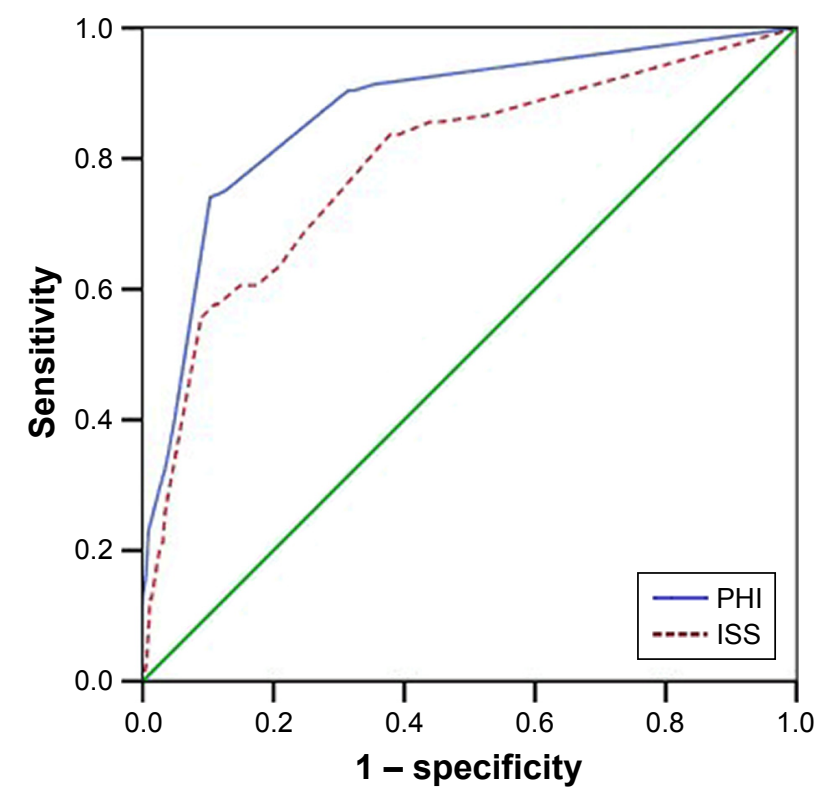

Figure I ROC curves of PHI and ISS in hospitalized patients with acute trauma. Abbreviations: ROC, receiver-operating characteristic; PHI, Prehospital Index; ISS, Injury Severity Score.

negative predictive value, positive likelihood ratio, and negative likelihood ratio were calculated for the PHI and ISS (Table 1). The sensitivity of PHI was superior to that of ISS $\left(\chi^{2}=6.975, P=0.008\right)$, but there was no significant difference in specificity $\left(\chi^{2}=1.907, P=0.167\right)$ or accuracy $\left(\chi^{2}=0.156, P=0.693\right)$.

\section{Comparison of death rate between mild- severity group and critical-severity group}

Based on the scoring criteria and optimal cutoff for the PHI, cases with PHI $<4$ were put into a mild-severity group $(1,510$ cases, with mortality of $1.72 \%)$ and those with PHI $\geq 4$ were put into a critical-severity group (292 cases, with mortality of $26.71 \%$ ). The mortality of the criticalseverity group was significantly higher than that of mildseverity group $\left(\chi^{2}=280.991, P<0.001\right)$. Based on the scoring criteria and optimal cutoff for the ISS, cases with ISS $<22$ were assigned to the mild-severity group $(1,554$ cases, with mortality of $2.83 \%$ ) and those with ISS $\geq 22$ were assigned to the critical-severity group (248 cases, with mortality of $24.19 \%)$. The mortality of the critical-severity group was significantly higher than that of the mild-severity group $\left(\chi^{2}=179.464, P<0.001\right)$.

\section{Discussion}

A fast, accurate, and standard condition assessment for patients with severe trauma is a great challenge in emergency rescue. Commonly used assessment tools include the Trauma and Injury Severity Score (TRISS) and a Severity Characterization of Trauma (ASCOT). Outcome prediction varies with the conditions of injury, response time, prehospital emergency rescue, medical care levels, and pretrauma diseases. China has not yet established a national trauma database, and the data required for the prediction are neither fully available nor accurate. ${ }^{13}$ Therefore, the prediction of survival simply based on TRISS and ASCOT may not be reasonable among Chinese patients.

The collection of data required for TRISS and ASCOT is time-consuming. Acute Physiology and Chronic Health Evaluation (APACHE) can offer a reliable assessment of trauma severity, ${ }^{4}$ but some problems still exist: APACHE applies to all intensive care unit patients, and may not be suitable for trauma patients; the acquisition of data for some parameters may take a long time, which affects the timeliness of assessment, and trauma patients usually undergo physiological disorders and constant changes. Frequent implementation of APACHE will increase not only pain and economic burden for patients but also the workload for medical staff. Moreover, patients may show low compliance with APACHE. The PHI can be determined easily within a short time, and has certain discriminating and assessing power for hospitalized patients with trauma.

The closer the ROC curve to the opportunity line (ie, the closer the $\mathrm{AUC}_{\mathrm{ROC}}$ to 0.5 ), the lower the discriminating power of the diagnostic indicator will be. The closer the $\mathrm{AUC}_{\mathrm{ROC}}$ value to 1 , the stronger the accuracy will be. It is generally believed that the diagnostic value is low when $\mathrm{AUC}_{\mathrm{ROC}}$ is $0.5-0.7$, moderate at $0.7-0.9$, and high above $0.9 .{ }^{14}$ In this study, we found that $\mathrm{AUC}_{\mathrm{ROC}}$ values for the PHI and ISS were 0.871 and 0.792 , respectively. These data indicate that the PHI has high discriminating power for potential critical illness among hospitalized patients with trauma and is superior to the ISS.

Table I Comparison of diagnostic evaluation indices of PHI and ISS

\begin{tabular}{lllllllllll}
\hline & Sen (\%) & Spe (\%) & FPR (\%) & FNR (\%) & Acc (\%) & $J^{*}$ & LR $^{+}$ & LR $^{-}$ & PV $^{+}$ & PV $^{-}$ \\
\hline PHI & 75.0 & 87.4 & 12.6 & 25.0 & 86.7 & 0.624 & 5.951 & 0.286 & 0.267 & 0.983 \\
ISS & 57.7 & 88.9 & 11.1 & 42.3 & 87.1 & 0.466 & 5.211 & 0.476 & 0.242 & 0.972 \\
\hline
\end{tabular}

Note: *Youden's index.

Abbreviations: PHI, Prehospital Index; ISS, Injury Severity Score; Sen, sensitivity; Spe, specificity; FPR, false-positive rate; FNR, false-negative rate; Acc, accuracy; LR, likelihood ratio; PV, predictive value. 
We performed a retrospective analysis based on test results, surgical records, and final diagnosis of included cases. The data from 1,802 hospitalized patients with trauma were collected. Among these patients, the mechanism of trauma was varied, such as traffic trauma, trauma related to work, burns, and accidental falls. In addition, males were dominant, because more males drive and work in industry in China than females. However, tolerance of trauma and responsiveness to treatment vary from one individual to another. Posttrauma physiological disturbance may not always correspond to the severity of anatomical injury, which leads to the diversity of clinical manifestations. Trauma patients usually undergo ultrasound and X-ray to determine severity after admission. However, of parenchymal viscera and neurovascular injuries cannot be diagnosed until surgery. In practice, the ISS may not be as accurate as expected. ISS scores are fixed for trauma patients with confirmed diagnosis, and thus cannot reflect the physiological disturbance dynamically. In spite of these advantages, the ISS reflects the severity of trauma and still applies to assessment and death prediction. Previous studies have shown that the ISS is an independent risk factor of death in trauma patients. ${ }^{15,16}$ In the present study, the $\mathrm{AUC}_{\mathrm{ROC}}$ was 0.792 for ISS, indicating moderate diagnostic value. The death rate was as high as $24.19 \%$ for cases with ISS $\geq 22$, and special care was required for these cases.

The PHI is determined based on such indices as systolic blood pressure, pulse rate, breathing state, and consciousness. The PHI is easy to operate, and has been widely applied as an ideal prehospital quantitative scoring method for trauma scenes. Mild severity is defined as PHI score of $0-3$, corresponding to zero death rate and surgical rate of $2 \%$. Critical severity is defined as PHI value of 4-20, corresponding to a death rate of $16.4 \%$ and surgical rate of $49.1 \%$. The optimal cutoff point for the PHI after admission was basically consistent with the value used for prehospital assessment. In our study, the death rate was $1.72 \%$ for PHI 0-3 and $26.71 \%$ for PHI 4-20. Some patients still had a $\mathrm{PHI} \geq 4$ after prehospital and nosocomial emergency-rescue measures, such as basic life support. A PHI score $\geq 4$ indicated failure to reverse physiological disturbance and the deterioration of patient conditions, so their mortality was far higher than prehospital mortality. However, patient characteristics may affect mortality, eg, sicker patients are more likely to be admitted and their mortality will be high. Therefore, the proportion of sicker patients in total patients enrolled in this study would have impacted the mortality association with either the ISS or PHI. This is one limitation of this study. Further prospective studies are needed to confirm our results. In addition, this was a single-center study, and biases could not be avoided. Multiple-center studies are necessary to test the prognostic value of the PHI in patients with trauma.

Comparison of the ISS and PHI showed that the sensitivity of the PHI was superior to the ISS, but there were no significant differences in specificity and accuracy between the two indicators. The PHI has a lower rate of missed diagnosis, and thus is more suitable for screening for potential critical illnesses at an earlier stage. Medical staff can provide extra care for acute-trauma patients with high PHI values.

In conclusion, the PHI is an easy, reliable, and objective outcome predictor for hospitalized patients with acute trauma. More importantly, the PHI can provide a timely and dynamic reflection of patient conditions. It is a useful tool to screen preliminarily for potential critical illnesses, and medical staff can get better preparation for the at-risk population.

\section{Acknowledgment}

This study was supported by the Specific Research Project of Health Pro Bono Sectors, Ministry of Health, China (01002014).

\section{Disclosure}

The authors report no conflicts of interest in this work.

\section{References}

1. Twijnstra MJ, Moons KG, Simmermacher RK, Leenen LP. Regional trauma system reduces mortality and changes admission rates: a before and after study. Ann Surg. 2010;251(2):339-343.

2. Spahn DR, Bouillon B, Cerny V, et al. Management of bleeding and coagulopathy following major trauma: an updated European guideline. Crit Care. 2013;17(2):R76.

3. Zhu Z, Shang X, Qi P, Ma S. Sex-based differences in outcomes after severe injury: an analysis of blunt trauma patients in China. Scand $J$ Trauma Resusc Emerg Med. 2017;25(1):47.

4. Frith D, Goslings JC, Gaarder C, et al. Definition and drivers of acute traumatic coagulopathy: clinical and experimental investigations. J Thromb Haemost. 2010;8(9):1919-1925.

5. Schöchl H, Nienaber U, Maegele M, et al. Transfusion in trauma: thromboelastometry-guided coagulation factor concentrate-based therapy versus standard fresh frozen plasma-based therapy. Crit Care. 2011;15(2):R83.

6. Schöchl H, Frietsch T, Pavelka M, Jámbor C. Hyperfibrinolysis after major trauma: differential diagnosis of lysis patterns and prognostic value of thrombelastometry. J Trauma. 2009;67(1):125-131.

7. Khan S, Davenport R, Raza I, et al. Damage control resuscitation using blood component therapy in standard doses has a limited effect on coagulopathy during trauma hemorrhage. Intensive Care Med. 2015; 41(2):239-247.

8. Moore EE, Knudson MM, Jurkovich GJ, Fildes JJ, Meredith JW. Emergency traumatologist or trauma and acute care surgeon: decision time. J Am Coll Surg. 2009;209(3):394-395.

9. Lansink KW, Gunning AC, Leenen LP. Cause of death and time of death distribution of trauma patients in a level I trauma centre in the Netherlands. Eur J Trauma Emerg Surg. 2013;39(4):375-383. 
10. Glance LG, Dick AW, Meredith JW, Mukamel DB. Variation in hospital complication rates and failure-to-rescue for trauma patients. Ann Surg. 2011;253(4):811-816.

11. Koehler JJ, Baer LJ, Malafa SA, Meindertsma MS, Navitskas NR, Huizenga JE. Prehospital Index: a scoring system for field triage of trauma victims. Ann Emerg Med. 1986;15(2):178-182.

12. Gennarelli TA, Wodzin E. AIS 2005: a contemporary injury scale. Injury. 2006;37(12):1083-1091.

13. Hannan EL, Mendeloff J, Farrell LS, Cayten CG, Murphy JG. Validation of TRISS and ASCOT using a non-MTOS trauma registry. J Trauma. 1995;38(1):83-88.
14. Gunning AC, Leenen LP. Applicability of the predictors of the historical trauma score in the present Dutch trauma population: modelling the TRISS predictors. J Trauma Acute Care Surg. 2014;77(4):614-619.

15. Abdelrahman H, El-Menyar A, Al-Thani H, et al. Time based traumarelated mortality patterns in a newly created trauma system. World $J$ Surg. 2014;38(11):2804-2812.

16. Morey AF. Re: External validation of a substratification of the American Association for the Surgery of Trauma renal injury scale for grade 4 injuries. J Urol. 2014;192(2):448-449.

\section{Publish your work in this journal}

Patient Preference and Adherence is an international, peer-reviewed, open access journal that focuses on the growing importance of patient preference and adherence throughout the therapeutic continuum. Patient satisfaction, acceptability, quality of life, compliance, persistence and their role in developing new therapeutic modalities and compounds to optimize clinical outcomes for existing disease states are major areas of interest for the journal. This journal has been accepted for indexing on PubMed Central. The manuscript management system is completely online and includes a very quick and fair peer-review system, which is all easy to use. Visit http://www. dovepress.com/testimonials.php to read real quotes from published authors.

\footnotetext{
Submit your manuscript here: http://www.dovepress.com/patient-preference-and-adherence-journal
} 